\title{
Non-Invasive RF Built-in Testing Using On-chip Temperature Sensors
}

\author{
E. Aldrete, M. Onabajo*, J. Altet, D. Mateo, J. Silva-Martinez* \\ Universitat Politècnica de Catalunya, Spain \\ * Texas A\&M University, USA
}

\begin{abstract}
This poster shows how to efficiently observe highfrequency figures of merit in $R F$ circuits by measuring $D C$ temperature with CMOS-compatible built-in sensors.
\end{abstract}

\section{Introduction}

In conventional built-in test (BIT) strategies for transceiver chains, power detectors are strategically placed at observation nodes of the circuits under characterization to measure the signal power along the path (e.g. [1]), easing the pass-fail testing as well as enabling the use of embedded active knobs in self-healing schemes for enhanced system performance. However, the finite input impedance of invasive detectors degrades the performance of the system.

In this poster, we demonstrate that it is feasible to use DC temperature measurements performed with built-in sensors placed near the circuit under test (CUT) to observe its high-frequency figures of merit.

\section{Results and discussion}

The designed on-chip characterization setup consists of a single-ended cascode low-noise amplifier (LNA) with inductive source degeneration [2] together with a differential temperature sensor [3].

The die micrograph is shown in Fig. 1 (TSMC $0.25 \mu \mathrm{m}$ CMOS, $1.25 \times 1.25 \mathrm{~mm}^{2}$ ). The locations of input transistor MNA, cascode transistor MNC, and temperature sensor are annotated. The built-in sensor provides a voltage proportional to the difference of temperature between two $15 \times 15 \mu \mathrm{m}^{2}$ sensing devices QS1 and QS2, which are spaced by $400 \mu \mathrm{m}$ to measure the difference between the test point and an ambient reference point. This sensor configuration provides a DC electrical signal proportional to the DC power dissipated by MNC. When multiple test points are monitored in the transceiver chain, the small sensing devices can be multiplexed to a single $0.004 \mathrm{~mm}^{2}$ sensor core that consumes a power of $15 \mu \mathrm{W}$.

The relationship between the electrical signals of a device and its power dissipation is dominated by the Joule effect that follows a quadratic law. Therefore, the DC power dissipated by MNC depends on both its DC bias and AC power at the signal frequency $f$, which is a function of the LNA gain and transconductance of MNA. By utilizing thermoelectric coupling, output power and linearity parameters can be determined from the observable temperature changes.

Fig. 1 compares the frequency response of the LNA with the DC temperature measurements obtained as a function of the LNA's input signal frequency. It can be observed that the LNA's center frequency corresponds to the maximum DC temperature increase. Monitoring figures of merit via temperature has two major advantages when compared with the traditional methods:

First, temperature sensors do not affect the signal path because they are not physically connected to the CUTs. Second, temperature measurements are performed at DC or low frequencies, even if the figure of merit to be measured is in the RF domain. This approach avoids the use of invasive high-frequency BIT circuitry and high-frequency equipment, reducing the complexity and cost of the external circuitry as well as simplifying the test setup.

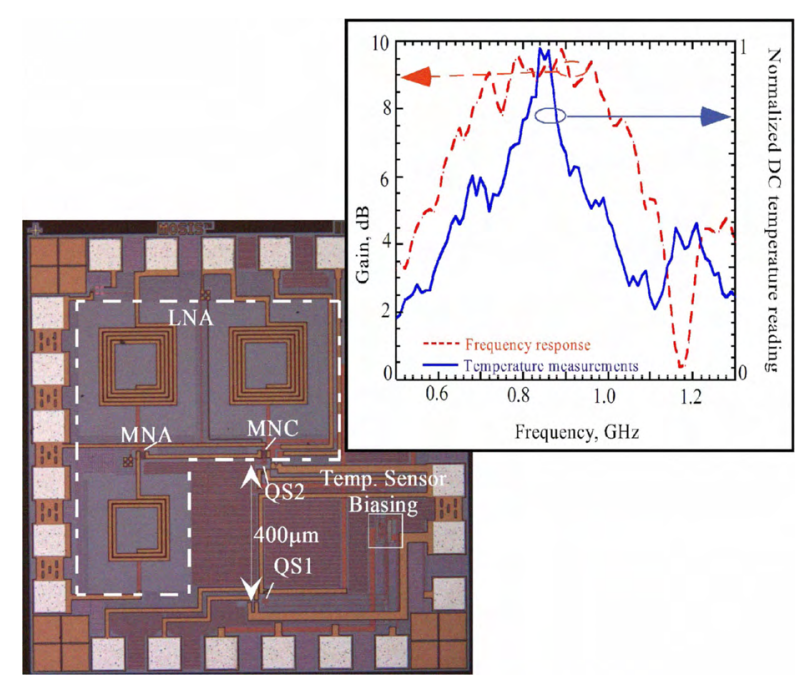

Fig. 1: Chip layout and measurements

\section{References}

[1] S. Bhattacharya and A. Chatterjee, "Use of embedded sensors for built-in-test of RF circuits," in Proc. IEEE Int. Test Conf. (ITC), pp. 801-809, Oct. 2004.

[2] D. K. Shaeffer and T. H. Lee, "A 1.5V, 1.5 GHz CMOS lownoise amplifier," IEEE J. Solid-State Circuits, vol. 32, no. 5, pp. 745-759, May 1997.

[3] J. Altet, et al., "Thermal coupling in integrated circuits: application to thermal testing", IEEE J. Solid-State Circuits, vol. 36, no. 1, pp. 81-91, Jan. 2001.

Acknowledgements: TEC2008-01856 